\title{
Janus kinase/signal transducer and activator of transcription inhibitors enhance the protective effect mediated by tanshinone IIA from hypoxic/ischemic injury in cardiac myocytes
}

\author{
MEI-QI ZHANG, JIAN-FENG TU, HUAN CHEN, YE SHEN, LING-XIAO PANG, \\ XIANG-HONG YANG, REN-HUA SUN and YUE-LIANG ZHENG \\ Department of Emergency, Zhejiang Provincial People's Hospital, Hangzhou, Zhejiang 310014, P.R. China
}

Received January 9, 2014; Accepted October 31, 2014

DOI: $10.3892 / \mathrm{mmr} .2014 .3063$

\begin{abstract}
Tanshinone IIA is a lipophilic abietane diterpene compound, which exhibits protective effects against ischaemia/reperfusion injury; however, the pathways responsible for the myocardial protective activities of tanshinone IIA remain to be elucidated. The aim of the present study was to investigate the effect of tanshinone IIA on the Janus-activated kinase (JAK)/signal transducer and activator of transcription (STAT) signaling pathway, which is associated with cardiac dysfunction during ischemia/reperfusion. The results demonstrated that tanshinone IIA protected myocardial cells from hypoxia/ischemia-induced injury in vitro and recovered decreased cell viability due to activation of the JAK2/STAT3 pathway, with $10 \mu \mathrm{M}$ tanshinone IIA exhibiting the most potent protective effects. Flow cytometric analysis revealed that tanshinone IIA reversed the apoptotic aggravation induced by JAK2/STAT3 inhibitors following hypoxic ischemia. However, JAK2 inhibitors promoted the myocardial protective effect of tanshinone IIA from hypoxic-ischemic injury. Furthermore, tanshinone IIA and JAK2/STAT3 inhibitors in combination augmented the protection of myocardial cells from apoptosis induced by ischemia/reperfusion preconditioning in vivo. In conclusion, the results of the present study indicated that JAK2/STAT3 inhibitors may enhance the protective effect of tanshinone IIA on cardiac myocytes from hypoxic ischemiainduced injury, therefore suggesting that JAK2/STAT3 inhibitors may have a potential application in combination therapies with tanshinone IIA.
\end{abstract}

Correspondence to: Dr Yue-Liang Zheng, Department of Emergency, Zhejiang Provincial People's Hospital, 158 Shangtang Road, Hangzhou, Zhejiang 310014, P.R. China E-mail: zhengyueliang1215@126.com

Key words: tanshinone IIA, hypoxic/ischemic injury, Janus kinase/signal transducer and activator of transcription

\section{Introduction}

Ischemic reperfusion (IR) triggers the disturbance of microcirculation, a primary cause of cardiac failure and mortality following cardiac surgeries $(1,2)$. Microcirculatory disturbances include endothelial cell injury, production of oxygen free radicals, energy supply reduction and myocardiocyte apoptosis (3).

Tanshinone IIA is an abundant lipophilic abietane diterpene compound which was isolated from Danshen (Salvia miltiorrhiza), a Traditional Chinese Medicine used for treating cardiovascular diseases $(4,5)$. In addition, tanshinone IIA was reported to balance the superoxide dismutase content and the malondialdehyde concentration in injured myocytes through scavenging free acids and reducing lipid peroxidation, which therefore protected myocytes and vascular endothelial cells during the IR process $(6,7)$. Furthermore, tanshinone IIA was found to markedly inhibit $\mathrm{H}_{2} \mathrm{O}_{2}$-induced oxidation in vitro, significantly inhibit IR-induced cardiomyocyte apoptosis via attenuation of morphological changes as well as reduce the percentage of terminal transferase 2'-deoxyuridine 5'-triphosphate (dUTP) nick end-labeling (TUNEL)-positive myocytes (8). However, it remains to be elucidated whether other signaling pathways are involved in the protective effects of tanshinone IIA on IR injury.

The Janus kinase/signal transducer and activator of transcription (JAK/STAT) signaling pathways regulate gene products involved in various cellular processes, including survival (9), apoptosis (10) and cell cycle progression (11); in addition, these pathways have been reported to have a vital role in inducing cardioprotection against IR injuries (10,12-15). Tanshinone IIA was found to inhibit JAK/STAT signaling and thereby induce apoptosis in cancer cells (16). The aim of the present study was to evaluate the effect of tanshinone IIA in combination with JAK2/STAT3 inhibitors on myocardial cells following the induction of hypoxic/ischemic injury in vitro and in vivo, as well as to elucidate the role of the JAK2/STAT3 signaling pathway in mediating the protective effects of tanshinone IIA.

\section{Materials and methods}

Cell culture and reagents. The $\mathrm{H} 9 \mathrm{c} 2$ embryonic rat heart-derived cells were purchased from the Chinese Academy 
of Sciences (Shanghai, China) cultured in Dulbecco's modified Eagle's medium (DMEM) supplemented with $10 \% \mathrm{v} / \mathrm{v}$ fetal bovine serum (FBS) and $100 \mathrm{mg} / \mathrm{ml}$ penicillin/streptomycin at $37^{\circ} \mathrm{C}$ in a humidified atmosphere containing $5 \% \mathrm{CO}_{2}$. Cells were maintained in serum-free culture and $100 \mathrm{nM}$ deferoxamine to induce ischemia and hypoxic conditions. DMEM, FBS, antibiotics and deferoxamine were purchased from Invitrogen Life Technologies, Carlsbad, CA, USA.

Animals. Male Sprague-Dawley rats (age, six weeks; weight, 200-220 g) were purchased from the Chinese Academy of Sciences (Shanghai, China). Rats were housed in a specific pathogen-free laboratory and exposed to a 12-h light/dark cycle at $37^{\circ} \mathrm{C}$ with free access to food and water. The rats were randomly divided into four groups (10 rats per group). Rats in control group were treated with phosphate-buffered saline, and rats in the experimental groups were administrated intraperitoneally with Tanshinone IIA, or Tanshinone IIA in combination with NSC74859 or AG490. Myocardial ischemia was induced by exposing the heart through a left thoracic incision and placing a 6-0 silk suture around the left coronary artery. Following $30 \mathrm{~min}$ of ischemia, the slipknot was released to allow reperfusion. At the end of the experiment, the rats were sacrificed using anesthetic (sodium pentobarbital, $30 \mathrm{mg} / \mathrm{kg}$ body weight; Akorn, Lake Forest, IL, USA).

Cytotoxicity assay. Cytotoxic effects of tanshinone IIA or JAK/STAT inhibitors (Sigma-Aldrich, St. Louis, MO, USA) on H9c2 cells were evaluated using a Cell Counting Kit 8 (CCK-8; Dojindo, Kumamoto, Japan). Cells were seeded onto 96-well microplates at a density of $2 \times 10^{4}$ cells per well, preconditioned with hypoxia/ischemia (hypoxia, $5 \% \mathrm{O}_{2}$ ) for 24 or $48 \mathrm{~h}$ and exposed to various concentrations of tanshinone IIA ( 0 , 2.5, 10 and $40 \mu \mathrm{M})$ or JAK/STAT inhibitors AG490 and NSC74859 (50 and $100 \mu \mathrm{M}$, respectively) for $12 \mathrm{~h}$. Cells were then incubated with CCK- 8 solution at $37^{\circ} \mathrm{C}$ for $3 \mathrm{~h}$. Optical density (OD) was measured at $450 \mathrm{~nm}$ using a MRX II microplate reader (Dynex Technologies, Inc., Chantilly, VA, USA). Cell viability was calculated as the percentage of viable cells in the drug-treated group versus that of the untreated control.

Flow cytometric analysis. Following treatment as above, cells $\left(>1 \times 10^{6}\right)$ were digested with $0.2 \%$ trypsin (BD Biosciences, Franklin Lakes, NJ, USA) and collected by centrifugation. Following washing twice with ice-cold phosphate-buffered saline, $10 \mu \mathrm{l}$ Annexin V-fluorescein isothiocyanate and $5 \mu \mathrm{l}$ propidium iodide (BD Biosciences) were added to a $85-\mu 1$ cell suspension and incubated for $15 \mathrm{~min}$ in the dark. Stained cells were then analyzed by flow cytometry using a FACS Calibur system with Cell Quest software version 5.1 (BD Biosciences).

Western blot analysis. Following treatment as above, cells were homogenized in lysis buffer containing $50 \mathrm{mmol} / \mathrm{l}$ Tris- $\mathrm{HCl}$ (pH 7.3), $150 \mathrm{mmol} / \mathrm{l} \mathrm{NaCl}, 5 \mathrm{mmol} / \mathrm{l}$ EDTA, $1 \mathrm{mmol} / 1$ dithiothreitol, $1 \%$ Triton X-100 and $1 \%$ protease inhibitor cocktail (Sigma-Aldrich). The lysates were centrifuged for $15 \mathrm{~min}$ at $12,000 \times \mathrm{g}$ and the resulting supernatant was transferred to a new tube and stored at $-70^{\circ} \mathrm{C}$. Protein concentrations were determined using a Bradford protein assay kit (Sigma-Aldrich) and proteins were separated using electrophoresis using $12 \%$
SDS-PAGE and transferred to nitrocellulose membranes (Sigma-Aldrich). The membranes were blocked for $1 \mathrm{~h}$ in Tris-buffered saline and Tween 20 (TBST; pH 7.6; SigmaAldrich) with 5\% non-fat dry milk and then incubated overnight at $4{ }^{\circ} \mathrm{C}$ with anti-mouse monoclonal antibodies against phospho-JAK2, phospho-STAT3, JAK2, STAT3 (dilution, 1:500) or $\beta$-actin (dilution, 1:1,000) purchased from Cell Signaling Technologies, Inc. (Beverly, MA, USA) followed by washes with TBST. The membranes were then probed with appropriate secondary antibodies (dilution, 1:5,000) at room temperature for $90 \mathrm{~min}$, followed by washes with TBST. Protein bands were detected using a chemiluminescence kit (Sigma-Aldrich) and were quantified using the Quantity One software package (version 4.6.2; Bio-Rad Laboratories, UK). The result of the control group was defined as $100 \%$.

In situ quantification of apoptotic cardiomyocytes. A portion of the myocardium from the mid-left ventricle of the middle slices was fixed in $4 \%$ formalin (Sigma-Aldrich). The apoptotic rate of the myocardial cells was analyzed using terminal deoxynucleotidyl transferase dUTP nick end labeling (TUNEL) staining using an in situ cell death detection kit (Roche, Basel, Switzerland). A double-staining technique was used; TUNEL staining was used to quantify apoptotic cell nuclei and DAPI staining (Sigma-Aldrich) was used to quantify the total myocardial cell nuclei. TUNEL-positive cells with green nuclear staining and all cells with blue nuclear DAPI staining were counted within five randomly selected fields under high-power magnification (DM-2500; Leica Microsystems, Wetzlar, Germany). The index of apoptosis was expressed as the ratio of positively stained apoptotic myocytes to the total number of myocytes counted $\times 100 \%$.

Histomorphological analysis. Following experimental preconditioning and measurement of hemodynamics, the hearts of mice were quickly removed and dissected into transverse slices from apex to base and processed for histology. Subsequently, the hearts were fixed in a $10 \%$ formalin solution (Sigma-Aldrich) for $24 \mathrm{~h}$, embedded in paraffin wax (SigmaAldrich). After deparrafinization with xylene, the wax was serially sectioned at $7 \mu \mathrm{m}$ using routine protocols (17). For the histomorphological analysis, the sections were stained with hematoxylin-eosin (HE; Sigma-Aldrich) and examined using a light microscope (BX40; Olympus, Tokyo, Japan).

Statistical analysis. Values are presented as the mean \pm standard error of the mean. Group comparisons were performed using analysis of variance with SPSS 13.0 software (SPSS, Inc. Chicago, IL, USA). P $<0.05$ was considered to indicate a statistically significant difference between values.

\section{Results}

Tanshinone IIA protects $\mathrm{H} 9 \mathrm{c} 2$ rat myoblast cells from hypoxic ischemia-induced injury and reduced viability. In order to determine the effect of tanshinone IIA on H9c2 cells, the H9c2 rat myoblast cell line was preconditioned with hypoxia/ischemia (24 and $48 \mathrm{~h}$ ) and treated with various concentrations $(0,2.5,10$ or $40 \mu \mathrm{M})$ of tanshinone IIA for $12 \mathrm{~h}$. A CCK8 assay was then performed (Fig. 1A and B). 
A
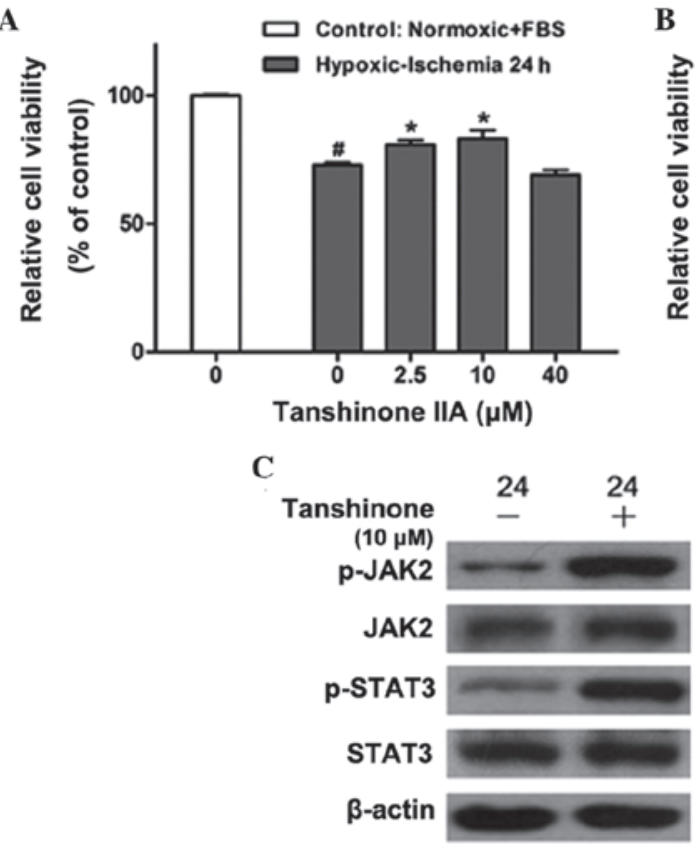
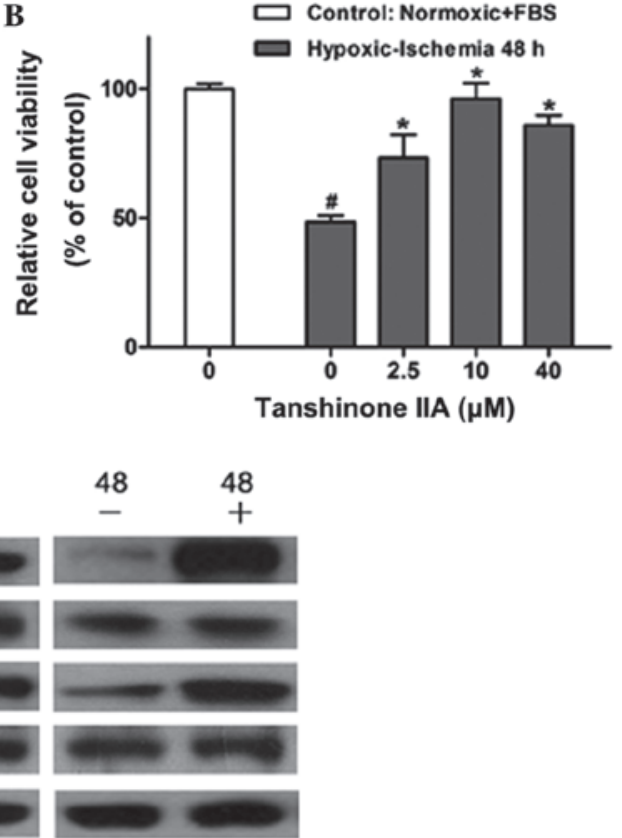

Figure 1. Effects of tanshinone IIA on cell viability and activation of the JAK/STAT pathway in the H9c2 rat myoblast cell line following hypoxic-ischemia injury. CCK- 8 assays were used to determine the dose-dependent effects of tanshinone IIA $(0,2.5,10$ and $40 \mu \mathrm{M})$ on the viability of the H9c2 cell line with or without hypoxic-ischemia pre-treatment for (A) $24 \mathrm{~h}$ and (B) $48 \mathrm{~h}$ as determined by the CCK- 8 assay. ${ }^{~} \mathrm{P}<0.05$ vs. control, ${ }^{*} \mathrm{P}<0.05$ vs. $0 \mu \mathrm{M}$ tanshinone IIA. (C) Western blot analysis of JAK2, p-JAK2, STAT3 and p-STAT3 proteins in control group and H9c2 cells treated with $10 \mu \mathrm{M}$ tanshinone IIA following hypoxic-ischemia pre-treatment for 24 and $48 \mathrm{~h}$. Each experiment was performed in triplicate. Values are expressed as the mean \pm standard deviation. $\beta$-actin was used as an internal control. CCK-8, cell counting kit 8; FBS, fetal bovine serum; JAK2, Janus kinase 2; STAT3, signal transducer and activator of transcription 3; p-, phosphorylated.

$\mathbf{A}$

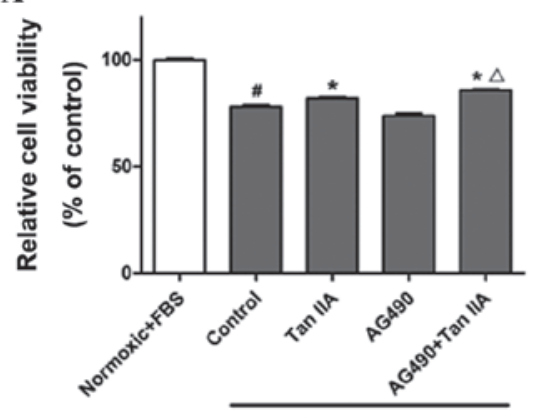

$\overline{\text { Hypoxic+Ischemia } 24 \mathrm{~h}}$

D

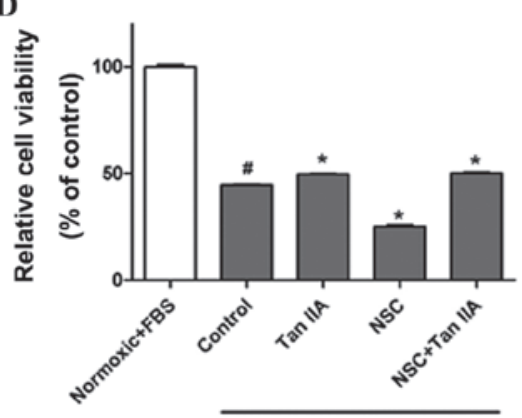

B

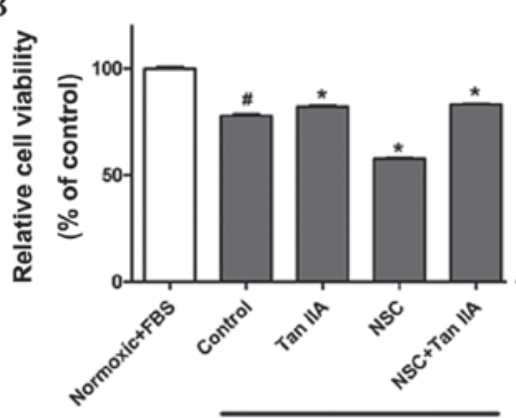

$\overline{\text { Hypoxic+Ischemia } 24 \mathrm{~h}}$

E

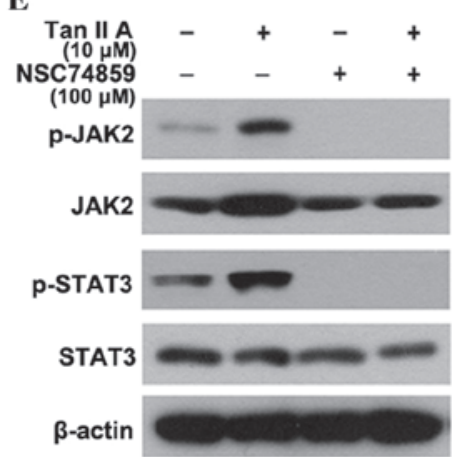

C

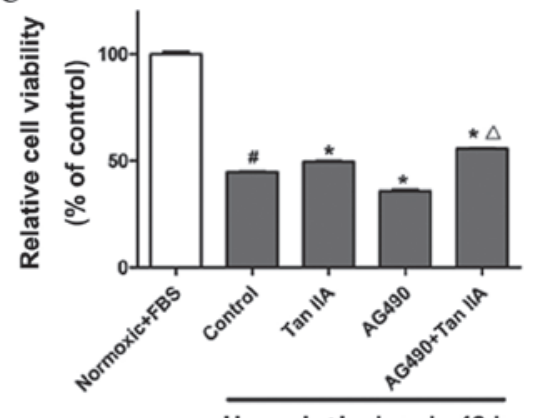

Hypoxic+Ischemia $48 \mathrm{~h}$

$\mathbf{F}$

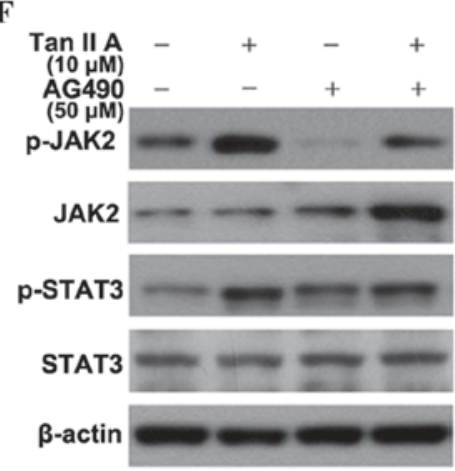

Figure 2. JAK2/STAT3 inhibitors reduce viability of H9c2 cells and promote the myocardial protective effects of tanshinone IIA against hypoxic ischemia. CCK-8 assays were used to determine cell viability in $\mathrm{H} 9 \mathrm{c} 2$ cells treated with $10 \mu \mathrm{M}$ tanshinone IIA alone or in combination with (A and C) JAK2 inhibitor AG490 or (B and D) STAT3 inhibitor NSC74859 following hypoxic-ischemia treatment for 24 or $48 \mathrm{~h}$, respectively. ${ }^{\text {} P}<0.05$ vs. normoxic + FBS group, ${ }^{\text {P }}<0.05$ vs. control group, ${ }^{\triangle} \mathrm{P}<0.05$ vs. Tan IIA group. (E and F) Western blot analysis of the protein expression levels of JAK2, p-JAK2, STAT3 and p-STAT3 following treatment with $10 \mu \mathrm{M}$ tanshinone IIA alone or in combination with (E) $100 \mu \mathrm{M}$ NSC74859 or (F) $50 \mu \mathrm{M}$ AG490 following hypoxic-ischemia treatment for $48 \mathrm{~h}$. Each experiment was performed in triplicate. Values are expressed as the mean \pm standard deviation. $\beta$-actin was used as an internal control. FBS, fetal bovine serum; Tan IIA, tanshinone IIA treatment; NSC, NSC74859 treatment; JAK2, Janus kinase 2; STAT3, signal transducer and activator of transcription 3; p-, phosphorylated; CCK-8, cell counting kit 8 . 
A
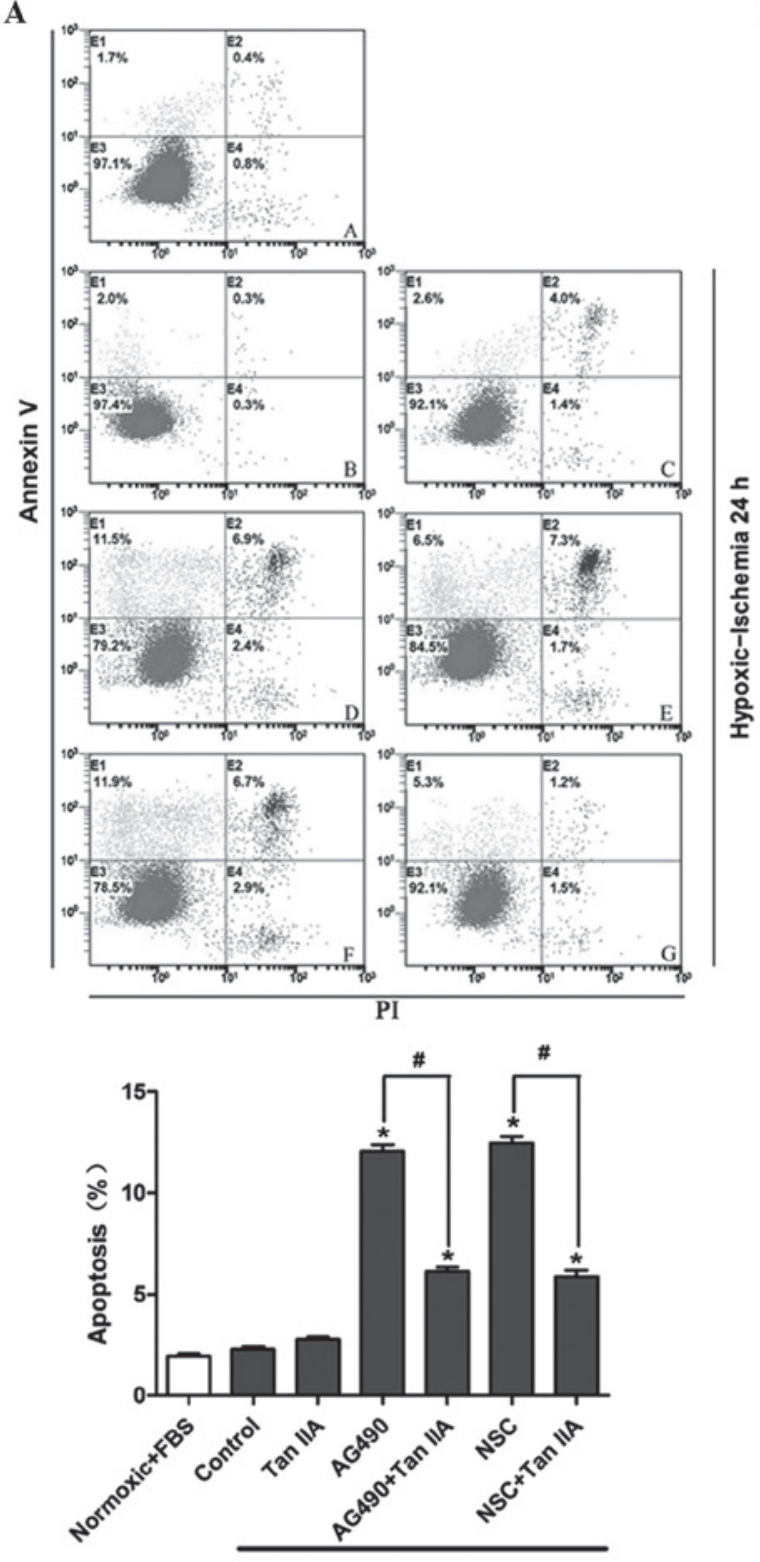

Hypoxic+lschemia $24 \mathrm{~h}$
B
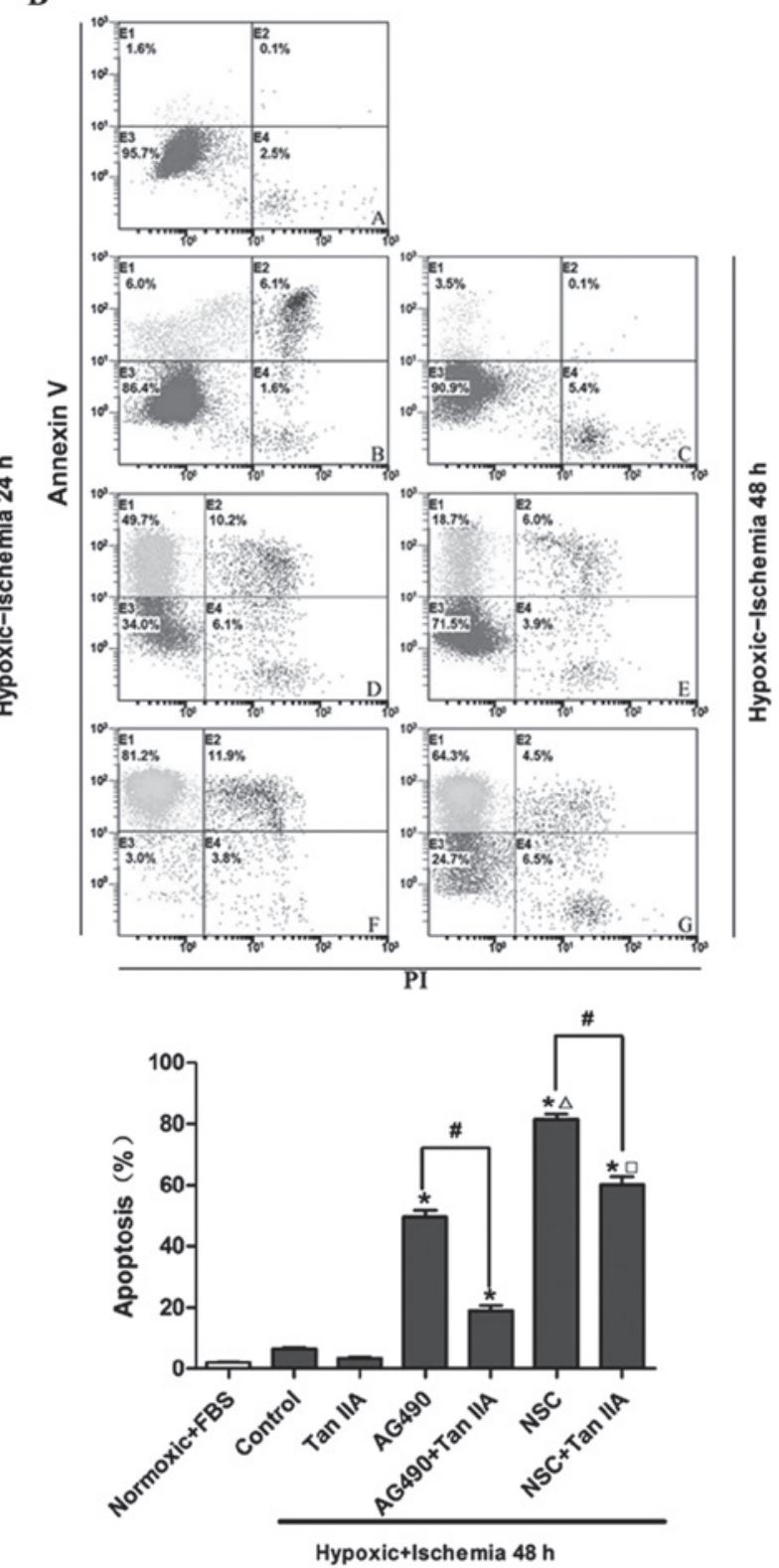

Figure 3. Tanshinone IIA inhibits apoptosis induced by JAK2/STAT3 inhibitors. Flow cytometric analysis of apoptosis in H9c2 cells post hypoxic ischemia preconditioning for (A) $24 \mathrm{~h}$ and (B) $48 \mathrm{~h}$, followed by determination of the apoptotic rates of cells treated with $10 \mu \mathrm{M}$ tanshinone IIA alone or in combination with the JAK2 inhibitor AG490 or the signal transducer and activator of transcription 3 inhibitor NSC74859. Each experiment was performed in triplicate. Values are expressed as the mean \pm standard deviation. ${ }^{*} \mathrm{P}<0.05$ vs. Tan IIA group, ${ }^{\#} \mathrm{P}<0.05$ between indicated groups, ${ }^{\Delta} \mathrm{P}<0.05$ vs. AG490 group; ${ }^{口} \mathrm{P}<0.05$ vs. AG490 + Tan IIA group. PI, propidium iodide; FBS, fetal bovine serum; Tan IIA, tanshinone IIA treatment; NSC, NSC74859 treatment; JAK2, Janus kinase 2; STAT3, signal transducer and activator of transcription 3.

Hypoxic/ischemic injury significantly reduced the viability of H9c2 cells; however, following treatment with tanshinone IIA, cell viability was markedly increased in a dose-dependent manner compared with that of the untreated group, with the most potent protective effects observed with $10 \mu \mathrm{M}$ tanshinone IIA. Furthermore, the protective effects of tanshinone IIA were more prominent following $48 \mathrm{~h}$ hypoxic/ischemia preconditioning compared to those in the group preconditioned for $24 \mathrm{~h}$.

Tanshinone IIA activates the JAK2/STAT3 pathway in $H 9 c 2$ cells. The JAK-STAT pathway is activated by stress $(9,18)$, including hypoxic/ischemic injury, which was first confirmed in a rodent model study (19). In order to verify whether the protective mechanisms of tanshinone IIA proceeded via the JAK2/STAT3 signaling pathway, the effects of tanshinone IIA on JAK2 and STAT3 activation were examined using western blot analysis. As shown in Fig. 1C, $10 \mu \mathrm{M}$ tanshinone IIA significantly upregulated the phosphorylation of JAK2 and STAT3 following 24 and 48 h of hypoxia/ischemia treatment, which was consistent with the results of the CCK-8 assay above.

JAK2 inhibitors promote the myocardial protective effects of tanshinone IIA from hypoxic-ischemic injury. In order to further determine whether the JAK2/STAT3 signaling pathway was 
A

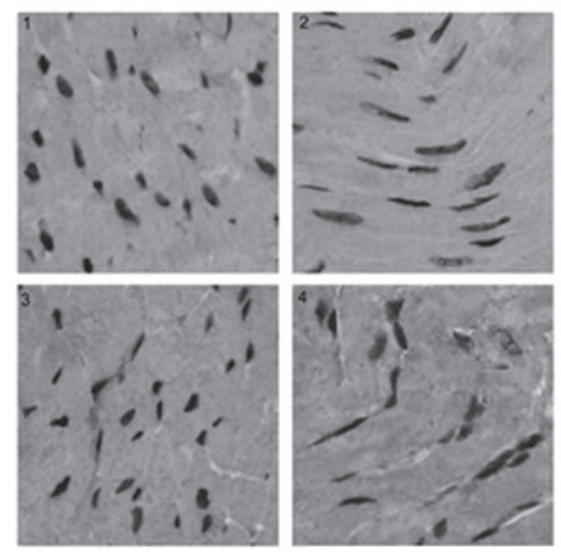

B

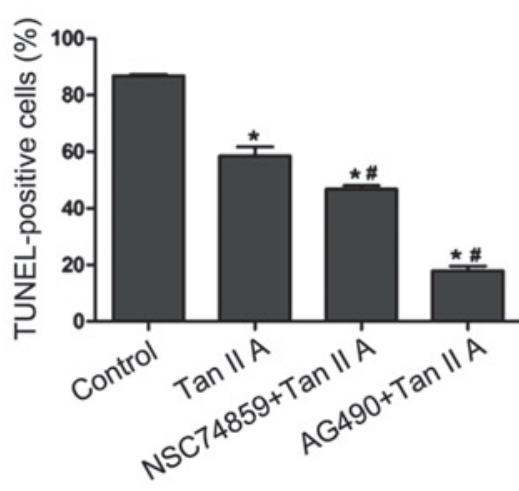

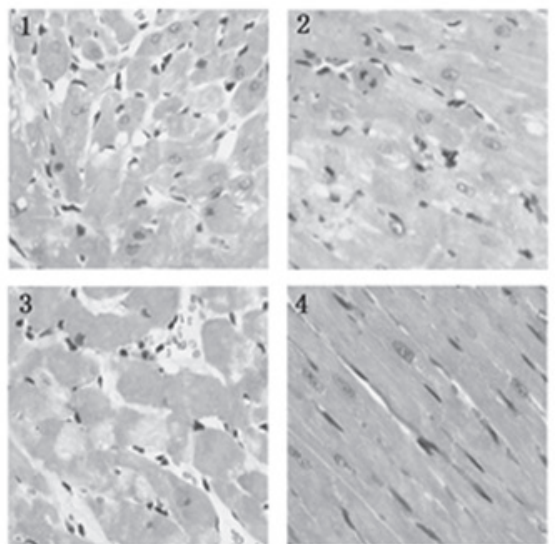

Figure 4. Representative slides for TUNEL and HE analyses of myocardial tissue of mice following hypoxic-ischemia preconditioning and treatment with $10 \mu \mathrm{M}$ Tan IIA alone or in combination with JAK2/STAT3 inhibitors. (A) Left: TUNEL staining in cardiomyocytes, nuclei with brown staining indicate TUNEL-positive cells (magnification, x200). Right: Quantitative results of TUNEL staining for each group. ${ }^{*} \mathrm{P}<0.05$ vs. control group, ${ }^{\sharp} \mathrm{P}<0.05$ vs. Tan IIA group. Values are expressed as the mean \pm standard deviation. (B) HE-stained myocardial samples (magnification, x200). Each experiment was performed in triplicate. 1, control group; 2, Tan IIA treatment group; 3, Tan IIA and JAK2 inhibitor AG490 treatment group; 4, Tan IIA and STAT3 inhibitor NSC74859 treatment group. TUNEL, terminal deoxynucleotidyl transferase 2'-deoxyuridine 5'-triphosphate nick end labeling; HE, hematoxylin-eosin; Tan IIA, tanshinone IIA; JAK2, Janus kinase 2; STAT3, signal transducer and activator of transcription 3.

responsible for the protective effect of tanshinone IIA, the JAK2 inhibitor AG490 (20) and the STAT3 inhibitor NSC74859 (17) were used to treat $\mathrm{H} 9 \mathrm{c} 2$ cells alone (50 and $100 \mu \mathrm{M}$, respectively) or in combination with $10 \mu \mathrm{M}$ tanshinone IIA. As shown in Fig. 2, the viability of $\mathrm{H} 9 \mathrm{c} 2$ cells was significantly reduced to $73 \pm 1.2 \%$ in the 24 -h hypoxic ischemia-preconditioned group (Fig. 2A and B) and $44 \pm 2.3 \%$ in the 48 -h hypoxic ischemiapreconditioned (Fig. 2C and D) compared to that of the normoxic and FBS groups $(\mathrm{P}<0.05)$. Tanshinone IIA reversed the injury and slightly restored cell viability to $80 \pm 1.1 \%$ and $49 \pm 1.4 \%$ in the groups pre-conditioned with 24 and $48 \mathrm{~h}$ hypoxic ischemia, respectively, which was significantly increased compared to that of the control group $(\mathrm{P}<0.05)$. Conversely, AG490 and NSC74859 reduced cell viability by $8 \pm 1.4 \%$ (Fig. 2A and C) and $13 \pm 2.7 \%$ (Fig. 2B and D) in the groups preconditioned with 24 and 48 h hypoxic ischemia, respectively, compared to that of the control group $(\mathrm{P}<0.05)$. Of note, co-administration of the JAK inhibitor AG490 with tanshinone IIA restored cell viability by $5.2 \pm 1.9 \%$ (Fig. $2 \mathrm{~A}$ and $\mathrm{C}$ ) in comparison with the tanshinone IIA only treatment group $(\mathrm{P}<0.05)$; however, no significant effects were observed following co-administration of tanshinone IIA with the STAT3 inhibitor NSC74859 (Fig. 2B and D). In addition, tanshinone IIA treatment significantly increased the phosphorylation level of JAK2 and STAT3. The level of p-JAK2 was inhibited following treatment with NSC74859 alone or NSC74859 combined with tanshinone IIA (Fig. 2E). As shown in Fig. 2F, AG490 suppressed the activation of JAK2 and STAT3; however, co-administration of tanshinone IIA and AG490 restored the phosphorylation level of JAK2 and STAT3. This therefore demonstrated that contrary to the expected result, the co-application of AG490 and tanshinone IIA did not eliminate the protective effect of tanshinone IIA, but further restored myocardial cell viability following the induction of hypoxic ischemia; furthermore, these results indicated that AG490 may affect the protective function of tanshinone IIA via inhibition of the JAK/STAT pathway.
Tanshinone IIA reverses the augmentation effect of JAK2/STAT3 inhibitors on apoptosis in H92C cells. Flow cytometric analysis was performed in order to investigate the effects of tanshinone IIA and JAK2/STAT3 inhibitors on hypoxic ischemia-induced cardiomyocyte apoptosis following 24 (Fig. 3A) and 48 h (Fig. 3B) of hypoxic ischemia preconditioning. Following treatment with the JAK inhibitor AG490, the percentage of hypoxic ischemia-induced apoptotic cells significantly increased from $2.0 \%$ to $11.5 \%$ and from 6.0 to $49.7 \%$ following 24 and $48 \mathrm{~h}$ of hypoxic ischemia preconditioning, respectively $(\mathrm{P}<0.05)$. Tanshinone IIA significantly reduced the exacerbation of hypoxic ischemia-induced apoptosis mediated by AG490 from 11.5 to $6.5 \%$ and from 49.7 to $18.7 \%$ following 24 and $48 \mathrm{~h}$ of hypoxic ischemia preconditioning, respectively $(\mathrm{P}<0.05)$. Similarly, the STAT3 inhibitor NSC74859 significantly increased the percentage of hypoxic ischemia-induced apoptotic cells from $2.0 \%$ to $11.9 \%$ and from 6.0 to $81.2 \%$ following 24 and $48 \mathrm{~h}$ of hypoxic ischemia preconditioning, respectively $(\mathrm{P}<0.05)$; however, co-administration of tanshinone IIA with NSC74859 markedly reduced the exacerbation of hypoxic ischemia-induced apoptosis from 11.5 to $5.3 \%$ and 81.2 to $64.3 \%$ following 24 and 48 h of hypoxic ischemia preconditioning, respectively $(\mathrm{P}<0.05)$.

Effect of tanshinone IIA on apoptosis. In order to verify the combined effects of tanshinone IIA and JAK/STAT inhibitors in vivo, tanshinone IIA and JAK/STAT inhibitors were administrated to normal mice following hypoxic/ischemia treatment. TUNEL-staining was used to identify apoptotic cells in the myocardium of control and treated mice (Fig. 4A). The number of TUNEL-positive cells observed was reduced in mice treated with tanshinone IIA; this effect was markedly enhanced in mice treated tanshinone IIA and AG490 combined $(\mathrm{P}<0.05)$. In addition, co-treatment with NSC74859 slightly decreased the percentage of TUNEL-positive cells compared with that in the tanshinone IIA group. 
JAK2 inhibitor enhances the ishemia/reperfusion injury alleviating effect of tanshinone IIA in vivo. In order to demonstrate the pathological effects of tanshinone IIA and JAK/STAT inhibitors on ischemia/reperfusion in vivo, mice underwent hypoxic-ischemia preconditioning and treatment with the respective drugs, then histological sections of the myocardium were obtained and stained with HE (Fig. 4B). Staining revealed that ischemia/reperfusion injury was markedly alleviated in the tanshinone IIA group compared with that in the control group; in addition, these beneficial effects were observed to be enhanced by co-treatment with AG490. However, no difference was observed between the ischemia/reperfusion injury in the tanshinone IIA group and the group threated with tanshinone IIA and NSC74859 combined.

\section{Discussion}

According to Traditional Chinese Medicine (TCM), tanshinone IIA, a derivative of phenanthrenequinone, enhances blood circulation and exhibits protective effects on the heart (4,21-23). Hypoxia/ischemia induces the disturbance of microcirculation, which leads to numerous adverse effects, including endothelial cell injury, energy supply reduction and myocardiocyte apoptosis (3). Previous studies have reported that tanshinone IIA markedly inhibited $\mathrm{H}_{2} \mathrm{O}_{2}$-induced cardiomyocyte apoptosis (24) as well as serum withdrawal- or ethanol- induced apoptosis in cultured PC12 cells (25). The results of the present study demonstrated that tanshinone IIA protected myocardial cells from hypoxia/ischemia-induced injury, as shown by CCK-8 assays and flow cytometric analysis; in addition, the effects of tanshinone IIA treatment were more marked following prolonged hypoxia/ischemia pretreatment from 24 to $48 \mathrm{~h}$.

The JAK-STAT pathway has previously been confirmed to be involved in cardiac apoptosis (26); as was first reported by a study on rat models of myocardial infarction, activation of the JAK1/STAT3 pathway limits apoptosis (27). The results of the present study suggested that tanshinone IIA treatment increased phosphorylation of JAK/STAT3, which may be the mechanism by which tanshinone IIA exerts its cardiomyocyte protective and antiapoptotic effects. Previous studies have demonstrated that activated STAT3 has an essential role in ischemic injury $(3,4)$; in addition, in these studies, the nonspecific JAK2 inhibitor AG490 and the specific STAT3 inhibitor NSC 74859 were administered in order to attenuate JAK/STAT3 activation prior to ischemic insult. AG-490 was reported to suppress the phosphorylation of STAT3, which resulted in increased caspase-3 activity and B cell lymphoma-associated $\mathrm{X}$ protein expression, concomitant with an increase in TUNEL-positive myocytes; this therefore indicated that JAK/STAT signaling may have antiapoptotic effects (28). Similarly, NSC74859 was reported to partially inhibit STAT3 dimerization and decrease target gene activation to reduce cell survival (29). In concurrence with these previous studies, the results of the present study demonstrated that AG490 and NSC74859 enhanced apoptosis and reduced cell viability following hypoxic/ischemic injury; in addition, tanshinone IIA reversed the exacerbation of hypoxia/ischemia-induced apoptosis mediated by JAK/STAT3 inhibitors.

Contrary to the expected results, co-administration of tanshinone IIA and JAK/STAT3 inhibitors augmented the myocardial protective function of tanshinone IIA against hypoxic/ischemic injury in $\mathrm{H} 9 \mathrm{c} 2$ cells in vitro as well as in mouse models in vivo. In addition, AG490 exhibited a more potent synergistic effect with tanshinone IIA than NSC74859. These results therefore suggested that the JAK/STAT3 signaling pathway had an important biphasic effect on the cardiac protection by tanshinone IIA from ischemia/reperfusion injury. One possible explanation for this is that JAK2 may activate STAT1 as well as STAT3; it has been previously reported that ischemia/reperfusion injury activated STAT1, which exerted a proapoptotic effect, whereas STAT3 had an antiapoptotic effect and antagonized the effects of STAT1 (30). This putative proapoptotic role of STAT1 in cardiomyocytes would be consistent with that observed in non-cardiac cells (31). In addition, the effects of tanshinone IIA may involve the STAT1 and STAT3 pathways, and the crosstalk between them determines the pharmacodynamic effects. It was previously reported that tanshinone IIA inhibited JAK2/STAT5 signaling, which induced apoptosis in cancer cells (16); in addition, AG920 was found to stimulate STAT5 signaling, which protected cells from apoptosis, therefore forming a negative feedback loop. Another possible explanation is that AG92, as a nonspecific inhibitor of JAK, initiated other pathways which were not involved in JAK2/STAT3 signaling. The exact mechanism underlying the effect of tanshinone IIA on ischemia/reperfusion remains elusive and further studies are required in order to elucidate the precise mechanisms by which JAK2/STAT3 inhibitors exert their synergistic action with tanshinone IIA. However, co-administration of JAK2/STAT3 pathway inhibitors and tanshinone IIA may be a potential novel treatment option for myocardial ischemia reperfusion in future clinical therapy.

In conclusion, the results of the present study demonstrated that JAK2/STAT3 inhibitors enhanced the protective effect of tanshinone IIA on cardiac myocytes following hypoxia/ischemia-induced injury, suggesting that JAK2/STAT3 inhibitors have the potential to be used for treating ischemia-reperfusion injury in combination with tanshinone IIA.

\section{Acknowledgements}

The present study was supported by general project funds of the Administration of Traditional Chinese Medicine of Zhejiang province (no. 2012ZA015).

\section{References}

1. Liao X, Wang L, Yang C, et al: Cyclooxygenase mediates cardioprotection of angiotensin-(1-7) against ischemia/reperfusion-induced injury through the inhibition of oxidative stress. Mol Med Rep 4: 1145-1150, 2011.

2. Ramzy D, Rao V and Weisel RD: Clinical applicability of preconditioning and postconditioning: the cardiothoracic surgeons's view. Cardiovasc Res 70: 174-180, 2006.

3. Wang N, Min X, Li D, He P and Zhao L: Geranylgeranylacetone protects against myocardial ischemia and reperfusion injury by inhibiting high-mobility group box 1 protein in rats. Mol Med Rep 5: 521-524, 2012.

4. Zhou L, Zuo Z and Chow MS: Danshen: an overview of its chemistry, pharmacology, pharmacokinetics, and clinical use. J Clin Pharm 45: 1345-1359, 2005.

5. Shang QH, Xu H, and Huang L: Tanshinone IIA: a promising natural cardioprotective agent. Evid Based Complement Alternat Med 7: 1120-1126, 2012. 
6. Luo Y, Xu, DQ, Dong HY, Zhang B, Liu Y, Niu W, Dong MQ and Li ZC: Tanshinone IIA inhibits hypoxia-induced pulmonary artery smooth muscle cell proliferation via Akt/Skp2/p27-associated pathway. PLoS One 8: e56774, 2013.

7. Fu J, Huang H, Liu J, Pi R, Chen J and Liu P: Tanshinone IIA protects cardiac myocytes against oxidative stresstriggered damage and apoptosis. Eur J Pharmacol 568: 213-221, 2007.

8. Zhou G, Jiang W, Zhao Y, et al: Sodium tanshinone IIA sulfonatemediates electron transfer reaction in rat heartmitochondria. Biochem Pharmacol 65: 51-57, 2003.

9. Koppikar P, Bhagwat N, Kilpivaara O, et al: Heterodimeric JAK-STAT activation as a mechanism of persistence to JAK2 inhibitor therapy. Nature 89: 155-159, 2012.

10. Zhang S, Liu X, Goldstein S, et al: Role of the JAK/STAT signaling pathway in the pathogenesis of acute myocardial infarction in rats and its effect on $\mathrm{NF}-\kappa \mathrm{B}$ expression. Mol Med Rep 7: 93-98, 2013.

11. Lange CM, Gouttenoire J, Duong FH, et al: Vitamin D receptor and Jak-STAT signaling crosstalk results in calcitriol-mediated increase of hepatocellular response to IFN- $\alpha$. J Immunol 192: 6037-6044, 2014.

12. Zgheib C, Kurdi M, Zouein FA, et al: Acyloxy nitroso compounds inhibit LIF signaling in endothelial cells and cardiac myocytes: evidence that STAT3 signaling is redox-sensitive. PLoS One 7: e43313, 2012.

13. Bolli R, Dawn B and Xuan YT: Role of the JAK-STAT pathway in protection against myocardial ischemia/reperfusion injury. Trends Cardiovasc Med 13: 72-79, 2003.

14. Yu HC, Qin HY, He F, et al: Canonical notch pathway protects hepatocytes from ischemia/reperfusion injury in mice by repressing reactive oxygen species production through JAK2/STAT3 signaling. Hepatology 54: 979-988, 2011.

15. Smith RM, Suleman N, Lacerda L, Opie LH, Akira S, Chien KR and Sack MN: Genetic depletion of cardiac myocyte STAT3 abolishes classical preconditioning. Cardiovasc Res 63: 611-616, 2004.

16. Jung JH, Kwon TR, Jeong SJ, et al: Apoptosis induced by Tanshinone IIA and Cryptotanshinone is mediated by distinct JAK/STAT3/5 and SHP1/2 signaling in chronic myeloid leukemia K562 cells. Evid Based Complement Alternat Med 2013: 805639, 2013.

17. Siddiquee K, Zhang S, Guida WC, et al: Selective chemica probe inhibitor of Stat3, identified through structure-based virtual screening, induces antitumor activity. Proc Natl Acad Sci USA 104: 7391-7396, 2007.

18. Mertens C and Darnell JE Jr: SnapShot: JAK-STAT signaling. Cell 131: 612-618, 2007.
19. Negoro S, Kunisada K, Tone E, et al: Activation of JAK/STAT pathway transduces cytoprotective signal in rat acute myocardial infarction. Cardiovasc Res 47: 797-805, 2000.

20. Grandage VL, Everington T, Linch DC and Khwaja A: Gö6976 is a potent inhibitor of the JAK 2 and FLT3 tyrosine kinases with significant activity in primary acute myeloid leukaemia cells. Br J Haematol 135: 303-316, 2006.

21. Chan P, Liu IM, Li YX, Yu WJ and Cheng JT: Antihypertension induced by tanshinone II A isolated from the roots of salvia miltiorrhiza. Evid Based Complement Alternat Med 12: 234-239, 2011.

22. Li YS, Wang ZH and Wang J: Effect of tanshinone IIA on angiotensin receptor in hypertrophic myocardium of rats with pressure over-loading. Chin J Integr Med 28: 632-636, 2008 (In Chinese)

23. Feng $\mathrm{J}$ and Zheng Z: Effect of sodium tanshinone IIA sulfonate on cardiac myocyte hypertrophy and its underlying mechanism. Chin J Integr Med 14: 197-201, 2008.

24. Yang R, Liu A, Ma X, et al: Sodium tanshinone IIA sulfonate protects cardiomyocytes against oxidative stress-mediated apoptosis through inhibiting JNK activation. J Cardiovasc Pharmacol 51: 396-401, 2008.

25. Meng XF, Zou XJ, Peng B, et al: Inhibition of ethanol-induced toxicity by tanshinone IIA in PC12 cells. Acta Pharmacol Sin 27: 659-664, 2006

26. Borensztejn A, Boissoneau E, Fernandez G, Agnès F and Pret AM: JAK/STAT autocontrol of ligand-producing cell number through apoptosis. Development 140: 195-204, 2013

27. Obana M, Maeda M, Takeda K, et al: Therapeutic activation of signal transducer and activator of transcription 3 by interleukin-11 ameliorates cardiac fibrosis after myocardial infarction. Circulation 121: 684-691, 2010.

28. Negoro S, Kunisada K, Tone E, et al: Activation of JAK/STAT pathway transduces cytoprotective signal in rat acute myocardial infarction. Cardiovasc Res 47: 797-805, 2000.

29. Siddiquee K, Zhang S, Guida WC, et al: Selective chemical probe inhibitor of Stat3, identified through structure-based virtual screening, induces antitumor activity. Proc Natl Acad Sci USA. 104: 7391-7396, 2007.

30. Stephanou A, Brar BK, Scarabelli TM, et al: Ischemia-induced STAT-1 expression and activation play a critical role in cardiomyocyteapoptosis. J Biol Chem 275: 10002-10008, 2000.

31. Kumar A, Kumar A, Michael P, et al: Human serum from patients with septic shock activates transcription factors STAT1, IRF1, and NF- $\mathrm{BB}$ and induces apoptosis in human cardiac myocytes. J Biol Chem 280: 42619-42626, 2005. 